\title{
Measurement of local strain and heat propagation during high-temperature testing in a split-Hopkinson tension bar system
}

\author{
V. Vilamosa ${ }^{1}$, A.H. Clausen ${ }^{1}$, O.S. Hopperstad ${ }^{1}$, T. Børvik ${ }^{1}$, and S. Skjervold ${ }^{2}$ \\ ${ }^{1}$ Structural Impact Laboratory (SIMLab), Centre of Research-based Innovation (CRI), Department of Structural \\ Engineering, Norwegian University and Science and Technology, 7491 Trondheim, Norway \\ ${ }^{2}$ Hydro Aluminium Extrusion Eurasia, Hydro Aluminium, Extrusion Competence Centre, 0240 Oslo, Norway
}

\begin{abstract}
Aluminium alloys are commonly used by the industry due to their good mechanical properties and their relatively low density. An accurate prediction of the behaviour of aluminium alloys under a wide range of temperatures and strain rates is important in numerical simulations of forming processes or applications involving adiabatic heating like penetration and crash situations. Several tests are needed at low, medium and high strain rates to study this behaviour. This paper will focus on the high strain rate test rig, which is a split- Hopkinson tension bar system (SHTB), the acquisition system for strain measurements, and a thermal analysis of the bars due to heating of the sample. A new way of doing local measurements with a high-speed camera will be presented. The thermal boundary conditions of the tests have been measured and simulated, and the results indicate that the stress wave propagation in the bars is not significantly affected by a local heating of the part of the bars which is closest to the sample.
\end{abstract}

\section{Introduction}

It is of increasing interest to enhance the knowledge on the mechanical behaviour of aluminium alloys. During both production and service, the material may be subjected to different strain rates and temperatures that can modify its physical and mechanical properties. The longterm aim of this project is to evaluate and model the interaction between temperature and strain-rate effects for the aluminium alloy AA6060. To establish an experimental database, several tests are performed at different strain rates using a conventional tension machine and a splitHopkinson tension bar system (SHTB).

This paper will focus on the SHTB test rig, the boundary conditions of the tests involving heating and the data acquisition. A high-speed camera is used to get local measurements of the strains after necking. This method gives an accurate measurement of the true strains in the necked section before and after the onset of necking as demonstrated by Hopperstad et al. [1]. The digital pictures also allow use of the Bridgman correction method that takes the triaxiality of the stress state at the neck into account.

The heating system used for the tests is an induction machine that facilitates a fast temperature increase in the specimen. Reaching the target temperature quickly is crucial to reduce the heat conduction to the bars in the SHTB test rig. Thermo-mechanical simulations of the sample and the bars have been carried out to evaluate the consequences of the heating of the bar. Finally, the results will be discussed and some directions will be given for further research.

\section{Experimental set-up for high strain-rate tests}

An introductory study on the aluminium alloy AA6060 applying the SHTB to be presented here has revealed that measurements of the true strains are difficult at high temperatures because the maximum stress and hence the necking point are reached at early stage, i.e strain level of about 0.2 . The only way to transcend this limit is to perform local measurements of the cross-section area of the sample. In order to do so, it was chosen to use a noncontact technique with a high-speed camera. The main advantage of such an approach is that it is not affected by the temperature of the sample, and it also provides the geometry of the notch, allowing use of the Bridgman correction.

\subsection{Strain measurement and stress acquisition}

\subsubsection{Engineering strain and stress measurements}

The SHTB consists of two bars, see Figure 1. Both the incident bar $\mathrm{ABC}$ and the transmission bar $\mathrm{DE}$ are made of Tibnor 52SiCrNi5 steel with a Young's modulus of $210 \mathrm{GPa}$ at room temperature.

During a test, the sample shown on Figure 2 is first screwed into the two bars (at $\mathrm{C}$ and $\mathrm{D}$ ). Thereafter, the incident bar is clamped at B and loaded in A. Thus, the part of the bar between A and B is stressed in tension. The force level is monitored by a pair of gauges (1). The tensile wave is then released instantaneously by removing the clamp at B. The incident and reflected strains are measured with a pair of strain gauges (2) while the transmitted wave is measured by the strain gauge pair (3), see Figure 1. The strain gauges (2) and (3) are located $600 \mathrm{~mm}$ from C and D, respectively. More details on the experimental set-up are provided by Chen et al. [2].

The nominal stress-strain curve from a typical AA6060 sample tested at room temperature and a strain-rate $\dot{\varepsilon}$ of 400 1/s is shown in Figure 3.

This curve is based on the classical formulas for analysis of results from Split-Hopkinson bar tests. Thus, the gauge readings of the reflected wave $\epsilon_{R}$ at (2) give the 


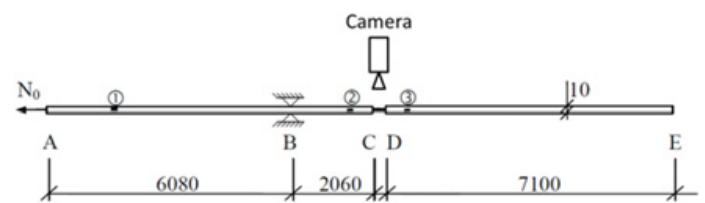

Fig. 1. SHTB and high speed camera.

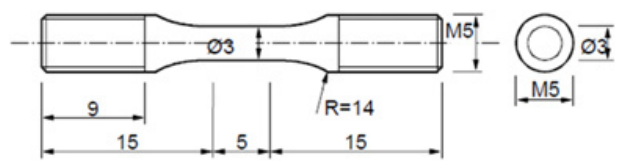

Fig. 2. Geometry of the samples.

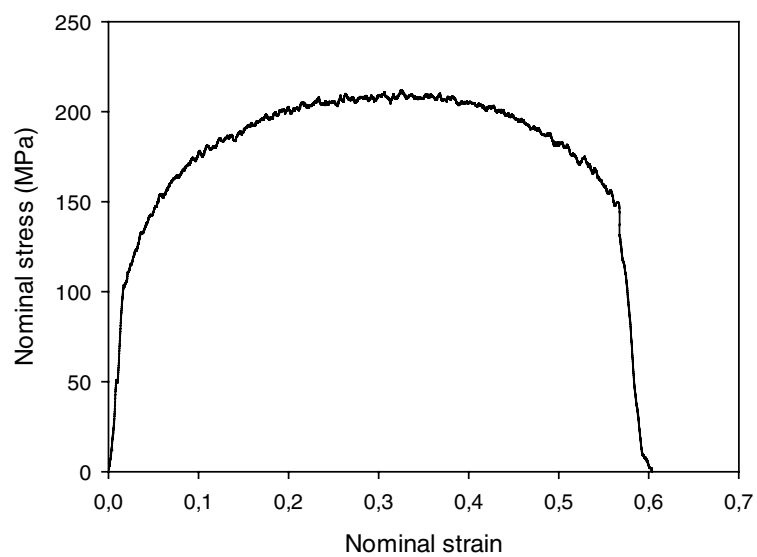

Fig. 3. Engineering stress-strain curve at $\dot{\varepsilon}=400 \mathrm{~s}^{-1}$ and $\mathrm{T}=$ $293 \mathrm{~K}$ (from gauges measurements).

nominal strain $\varepsilon$ and the strain of the transmitted wave $\epsilon_{T}$ at (3) is required for calculation of the nominal stress $\sigma$ :

$$
\begin{gathered}
\sigma=\frac{A_{b}}{A_{s}} E_{b} \varepsilon_{T} \\
\varepsilon=-2 \frac{C_{b}}{L_{s}} \int_{0}^{t} \varepsilon_{R} d t
\end{gathered}
$$

In these expressions, $E_{b}$ is the Young's modulus of the bars, $A_{b}$ and $A_{s}$ are respectively the cross-section area of the bars and the sample, $c_{b}$ is the longitudinal wave propagation velocity in the bars, and $L_{S}$ is the length of the specimen. Further, the nominal strain rate $\dot{\varepsilon}$ is given by:

$$
\dot{\varepsilon}=-2 \frac{C_{b}}{L_{S}} \varepsilon_{R}
$$

From Equations (1) and (2), it is possible to calculate the true strain $\varepsilon_{t}$ and true stress $\sigma_{t}$ until the onset of necking, applying the classical relations:

$$
\begin{gathered}
\sigma_{t}=\sigma(1+\varepsilon) \\
\varepsilon_{t}=\ln (1+\varepsilon)
\end{gathered}
$$

Equation (2), (4) and (5) assume that the strains are homogeneous through the gauge length $L_{s}$ of the specimen. Therefore, a local measurement of the strains in the neck is required after localisation occurs.

\subsubsection{Local measurements of the strain and stress}

During the entire test, a digital high-speed camera monitored the sample. The acquisition frequency was $50000 \mathrm{~Hz}$. The pictures served to give a local value of the true strains and stresses in the necked section by measuring the diameter of the specimen during the test. It was assumed that the cross-section remained circular. Further, it allowed for calculating the true stress. Triggered by the leading edge of the incoming stress wave at gauge (2), the camera was synchronised with the measured signal. Thus, the camera started recording before the strain wave reached the sample. In order to obtain a complete synchronisation of both signals, the time associated with the wave propagation has to be determined and subtracted from the running time of the camera. The distance between (2) and the sample is $600 \mathrm{~mm}$, and the celerity of the strain wave at room temperature is approximately $5100 \mathrm{~m} / \mathrm{s}$ in the bars. Thus, the stress wave takes $0.118 \mathrm{~s}$ to reach the sample after passing (2).

The sample was painted black to better determine its shape during deformation, see Figure 6. A Matlab script was written to measure the geometry of the sample during the test. Two white points were applied on the sample to get an additional measurement of the nominal strain $\varepsilon^{c}$ with the camera before necking by monitoring the distance $L$ at $t=0$ and the displacement $\Delta L$ between these two points:

$$
\varepsilon^{c}=\frac{\Delta}{L}
$$

The true strain from the camera $\varepsilon_{c}^{t}$ is calculated with Equation (5), applying the nominal strain from Equation (6) until the onset of necking. Assuming incompressible plastic deformation, $\varepsilon_{c}^{t}$ is calculated as the ratio between the diameter of the smallest section $D_{0}$ at $t=0$ and the diameter $D_{S}$, which is determined from the pictures taken during the test:

$$
\varepsilon_{t}^{c}=\ln \left(\frac{A_{0}}{A_{S}}\right)=2 \ln \left(\frac{D_{0}}{D_{S}}\right)
$$

where $A_{0}$ and $A_{S}$ are respectively the initial area of the section and the smallest section during the test. The true stress $\sigma_{t}^{c}$ is calculated, again applying the diameter measured from the pictures and assuming a circular shape of the cross-section during the entire test:

$$
\sigma_{t}^{c}=\sigma \frac{A_{0}}{A_{S}}
$$

Because different temperatures are applied, the paint put on the sample does not react in the same way for each test. The applied paint is a common paint that resists temperatures not exceeding $600 \mathrm{~K}$. A special thermal resistant paint is required for higher temperatures.

Due to the cracking of the paint at high temperatures, it was decided to use the bars measurements until necking and then to use the evolution of the smallest diameter to calculate the true strains in the sample using Equation (7). The true stress is calculated with Equation (8) during the entire test. 


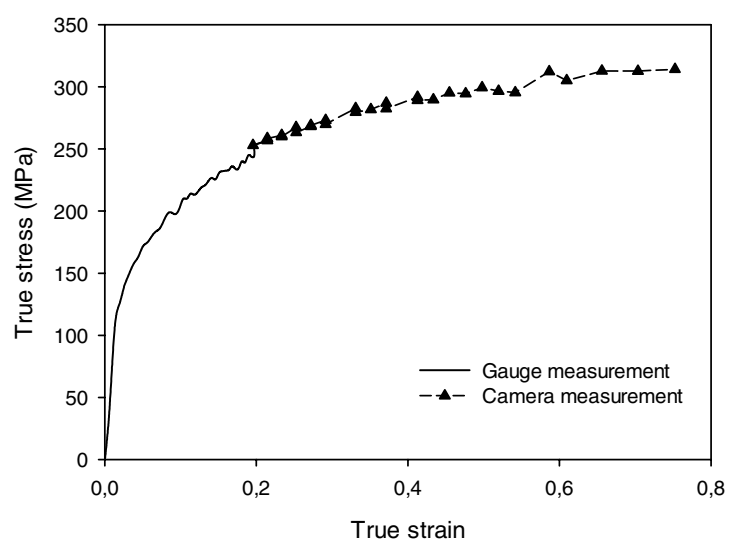

Fig. 4. True stress versus true strain curve based on measurement from the bars and camera $\left(\dot{\varepsilon}=400 \mathrm{~s}^{-1}, \mathrm{~T}=293 \mathrm{~K}\right)$.

As a first observation, it can be mentioned that the true strain range studied is much larger with the use of the high speed camera even for low temperature tests as shown in Figure 4.

\subsection{The Bridgman correction}

The digital pictures can be used to determine the shape of the neck during deformation. Therefore, it is possible to calculate a stress corrected with the Bridgman method $[3,4]$. This method uses the geometry of the notch to calculate a factor that can be applied on the true stress to get an equivalent stress valid for the multi-axial stress state after necking. Thus, the Bridgman correction is used to take the triaxiality of the stress field in the neck into account. tions:

The Bridgman correction is based on three assump-

- The strain distribution in the minimum cross-section should remain uniform.

- A grid line is deformed into a curve at the neck.

- The ratio of the principal stresses remains constant all along the test.

A corrector factor $k$ is calculated using the shape of the neck:

$$
k=\frac{1}{\left(1+\frac{2 R}{a}\right) \ln \left(1+\frac{a}{2 R}\right)}
$$

where $a$ is the radius of the smallest cross-section, and $R$ is the radius of curvature of the neck. Multiplied with the true stress $\sigma_{t}$ the factor $k$ gives an equivalent stress defined by:

$$
\sigma_{e q}=k \sigma_{t}
$$

The radial and axial stresses $\sigma_{r}$ and $\sigma_{a}$ as function of the distance $r$ to the symmetry axis can also be calculated:

$$
\begin{aligned}
& \sigma_{r}=k \sigma_{t} \ln \left(\frac{a^{2}+2 a R-r^{2}}{2 a R}\right) \\
& \sigma_{a}=k \sigma_{t}\left(1+\ln \left(\frac{a^{2}+2 a R-r^{2}}{2 a R}\right)\right)
\end{aligned}
$$

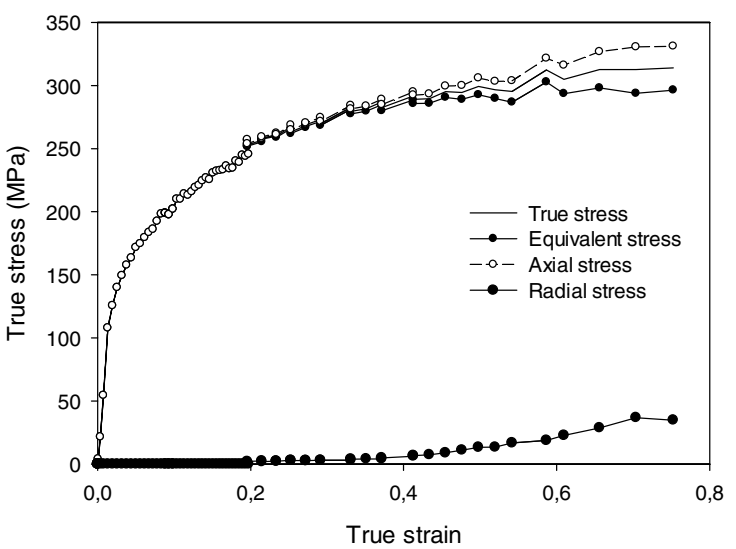

Fig. 5. True, equivalent, axial, and radial stresses in the centre of the sample at the minimum cross-section as a function of the true strain, $\dot{\varepsilon}=400 \mathrm{~s}^{-1}, \mathrm{~T}=293 \mathrm{~K}$ (gauges and camera measurement).

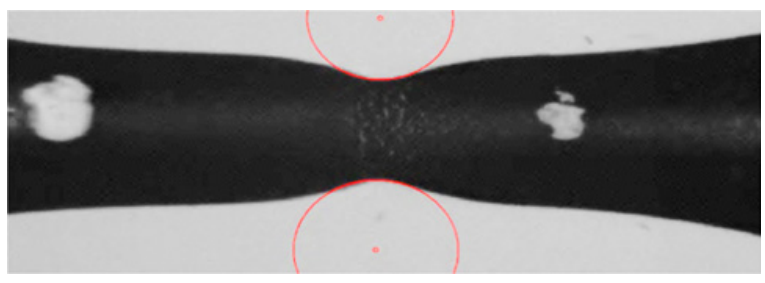

Fig. 6. Necking and radius of curvature calculation in Matlab.

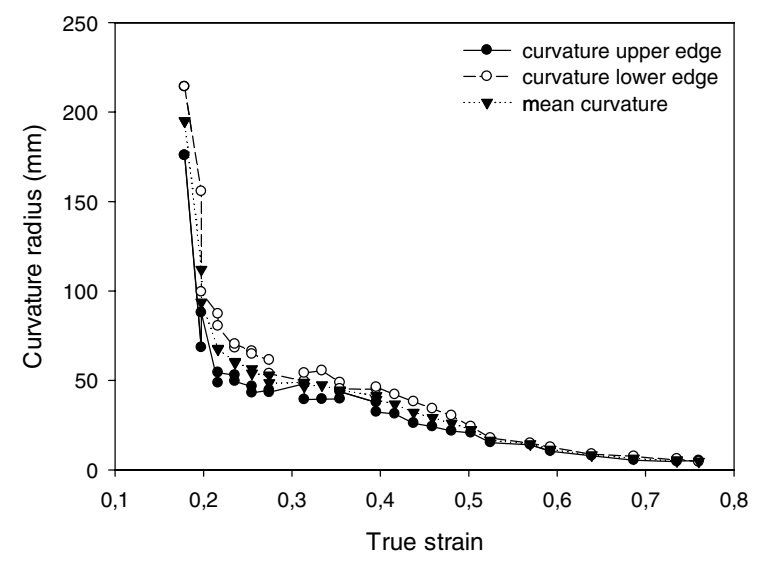

Fig. 7. Radius of curvature as function of true strain.

These stresses are shown on the Figure 5. For each frame, the radius of curvature for the upper and lower edges is calculated by a least squares method using Matlab. Figure 6 illustrates this approach at a certain deformation level. It appears from this particular photo that the predicted radius is somewhat larger at the lower surface of the sample.

Figure 7 presents the curvature radius from the upper and lower edge as function of the true strain. It shows that there are some differences at the beginning of the necking between the two radii of curvature, but they converge to the same value. These differences could be due to the resolution of the images, but also to the radius of curvature which can be 100 times greater than the distance of the analysed edge. Moreover, there might be a real difference in curvature between the upper and lower surface at the pictures due to the material anisotropy, see Figure 6. 


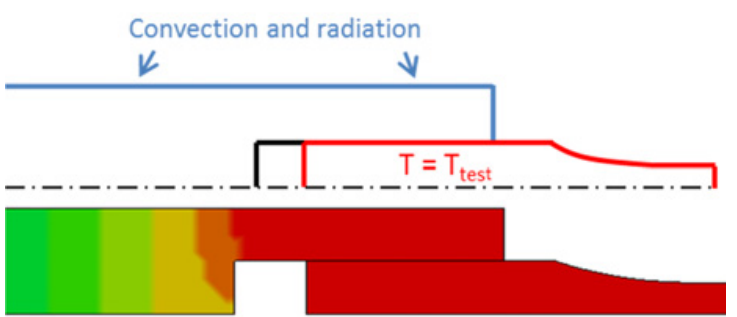

Fig. 8. Boundary conditions and temperature in the bar and sample during a simulation.

\section{Influence of the heat conduction from sample to bars}

\subsection{Thermal boundary conditions}

The aim of this thermal study was to determine the maximum temperature reached in the bars during the heating of the sample, and to simulate the influence of the temperature on the signals recorded by the strain gauges.

Firstly, some thermal tests were performed by heating the sample and recording the temperature with three thermocouples at distances of $10 \mathrm{~mm}, 50 \mathrm{~mm}$ and $100 \mathrm{~mm}$ from the sample. The temperature in the sample was monitored by a thermocouple as well, and it was compared with temperatures given by an infrared camera to ensure the accuracy of the measurement. In advance, the sample was painted black to get a constant emissivity, which is the main requirement for use of the thermal camera. The sample surface temperatures measured by the thermocouple and the thermal camera on the sample were in good agreement for temperatures lower than $600 \mathrm{~K}$. For higher temperatures, the emissivity of the sample changed because the paint burned. This resulted in a difference up to $45 \mathrm{~K}$ between the two thermal measurement tools.

Concerning the heat conduction into the bars, it was observed that the temperature measured by the thermocouple $10 \mathrm{~mm}$ distance from the end of the bar was very close to the temperature in the sample. A closer look at the heat conduction from the heated sample to the bars was therefore required.

A thermal analysis of the SHTB system was performed in LS-DYNA and compared with the thermal tests. The thermal analysis in LS-DYNA uses an implicit formulation. An axi-symmetric model of the steel bars and the aluminium sample was established where the nodes between the sample and the bars were merged. The heat flow between the two parts is governed by the thermal conductivity of steel and aluminium. Both materials are assumed to be thermally isotropic. The temperature is applied to the sample and the surfaces of the bars are submitted to the convection and radiation as shown in Figure 8 .

The measured temperature of the sample during a test was used as input in LS-DYNA and the temperature in the bars was thereafter fitted at each thermocouple location by adjusting the convection and radiation parameters in Table 1. Figure 9 shows that the numerical results are in good accordance with the test observations at all three positions instrumented with thermocouples. Davoodi et al.
Table 1. Thermal problem parameters.

\begin{tabular}{|c|c|c|c|}
\hline $\begin{array}{c}\text { Thermal } \\
\text { conductivity } \\
\text { of Tibnor } \\
\begin{array}{c}52 \text { SiCrNi5 } \\
\text { Steel } \\
{[\mathrm{W} /(\mathrm{mK})]}\end{array}\end{array}$ & $\begin{array}{c}\text { Thermal } \\
\text { conductivity } \\
\text { ofAA6060 } \\
{[\mathrm{W} /(\mathrm{mK})]}\end{array}$ & $\begin{array}{c}\text { Convection } \\
\text { from the } \\
\text { bars } \\
{\left[\mathrm{W} /\left(\mathrm{m}^{2} \mathrm{~K}\right)\right]}\end{array}$ & $\begin{array}{c}\text { Radiation } \\
\text { from the bars } \\
{\left[\mathrm{W} /\left(\mathrm{m}^{2} \mathrm{~K}^{4}\right)\right]}\end{array}$ \\
\hline 32 & 180 & 6 & $1 \cdot 10^{-7}$ \\
\hline
\end{tabular}

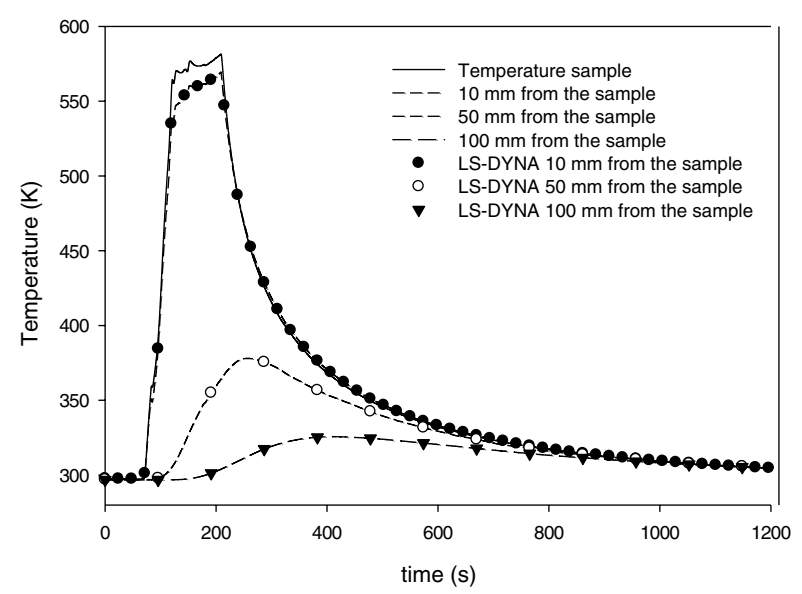

Fig. 9. Temperature in the bars and sample in the test and the simulation.

[5] have also found that the increase of temperature is significant only in the part of the bars which is closest to the sample. A relevant question is therefore whether this local temperature increase of the bars has any influence on the stress wave propagation and hence the application of Equations (1) and (3).

\subsection{Influence of the temperature in the bars}

A full thermo-mechanical simulation was conducted to investigate the influence of the temperature in the test. The analysis takes the variation of Young's modulus of the bars as function of temperature into account, see Figure 10. The relationship shown in this figure was established for a steel material having less than $0.6 \%$ of carbon [6]. A significant reduction appears for temperatures higher than $600 \mathrm{~K}$ and Young's modulus has decreased to $50 \%$ of the value at room temperature at $900 \mathrm{~K}$.

The material parameters used for the sample in these simulations were assumed to be independent of the temperature. This means that the behaviour of the sample is the same for all simulations and applied temperatures, serving to isolate the effect of the temperature in the bars.

The thermomechanical simulations are based on a coupled implicit-explicit calculation and use the same philosophy as described by Chen et al. [2]. The intension with the model is to measure the strains in the same way as it is done during a test. The first step in the numerical simulation is to heat the sample to a temperature of $780 \mathrm{~K}$ during a time of 20 seconds, which corresponds rather well with the conditions in a test. The temperature increase in the bars is calculated during the implicit thermal analysis part of the simulation. The clamp is then removed right 


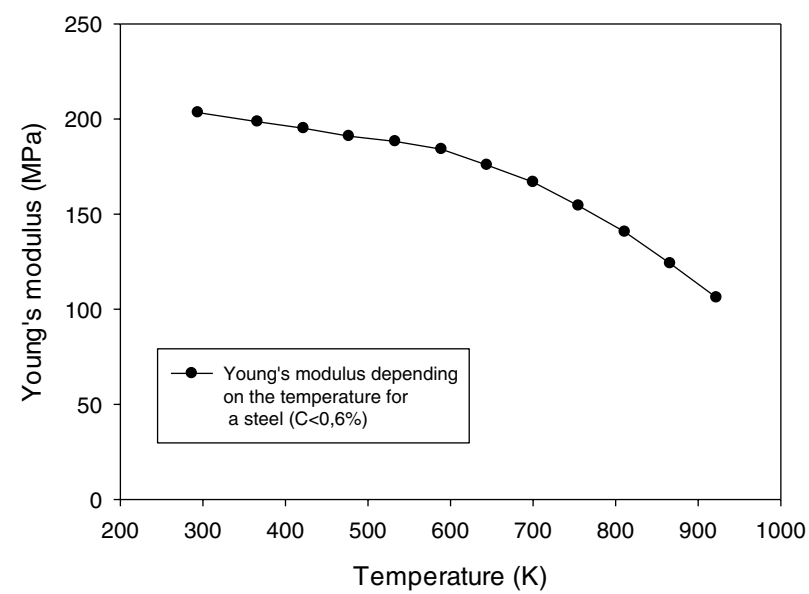

Fig. 10. Young's modulus for steel depending on the temperature.

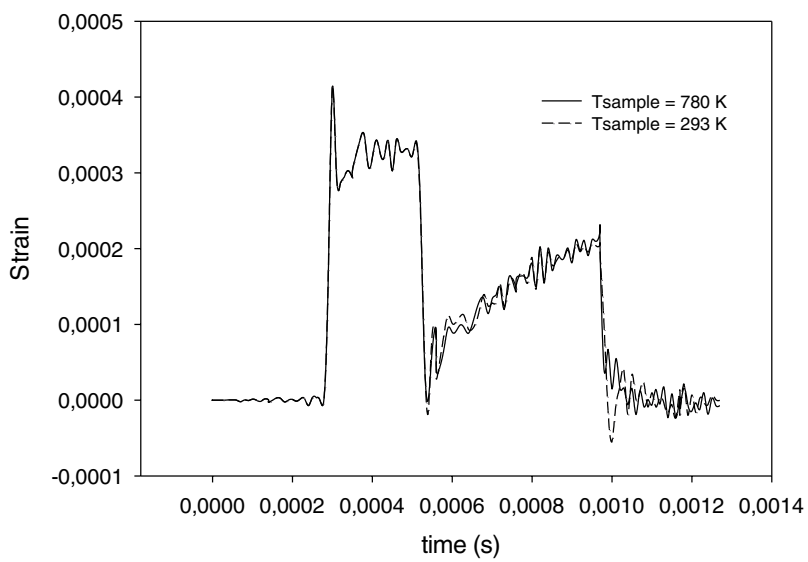

Fig. 11. Strain signal in gauge (2) for high and low temperature; $\dot{\varepsilon}=200 \mathrm{~s}^{-1}$.

after the switch from implicit to explicit analysis, and the mechanical part of the response from the test is then calculated. In addition, a test is simulated at room temperature.

The strains were measured at the same location in the model as in the real system (2) and (3)). Figure 11 shows that the reflected signal is almost the same for the two simulations. Neither any delay nor any change of the wave length were observed for the two applied temperatures. Therefore, the local heating of the parts of the bars that are close to the samples does not seem to have any influence on the signals picked up by the gauges. Consequently, the classical formulas in Eqs. (1)-(3) can be applied without any correction. Nevertheless, in future tests precautions will be taken to cool down the bars close to the sample.

\section{Conclusion}

An experimental approach which will be used to establish a data base for the AA6060 under the combined effects of high strain rates and temperatures has been evaluated in this paper. A local measurement of the deformed geometry of the sample is obtained with a high speed camera. This allows for calculation of the true strain and stress in an extended range. This technique provides the shape of the neck, and a subsequent use of the Bridgman correction allows for calculation of the equivalent stress in the neck.

The thermal boundary conditions have been checked and simulated to validate the measurement procedure. The temperature increase of the bars is significant close to the ends, but it is localised and does not affect the strain measurement in the bars. Despite the results, cooling will be used on the edges of the bars to ensure a high and homogeneous temperature in the sample.

\section{References}

1. O. Hopperstad, T. Børvik, M.Langseth, K. Labibes, C. Albertini, On the influence of stress triaxiality and strain rate on the behavior of a structural steel. PartI. Experiments, European Journal of Mechanics A/Solids 22, 1-13 (2003).

2. Y. Chen, A.H Clausen, O.S. Hopperstad, M. Langseth, Application of a split-Hopkinson tension bar in a mutual assessment of experimental test and numerical predictions,International Journal of Impact Engineering, 38, 824-836 (2011).

3. M. Gromada, G. Mishuris, A.Öchsner, Correction formulae of the Stress Distribution in Round Tensile Specimens at Neck Presence (Springer 2011).

4. G. Miroe, A new model for the elastoplastic characterization and the stress-strain determination on the necking section of a tensile specimen, International Journal and Structures 41, 3545-3564 (2004).

5. B. Davoodi, A. Gavrus, Eric. Ragneau, An experimental and numerical analysis of the $h$ eat problem in SHPB at elevated temperatures, Measurement Science and Technology, 16, 2101-2108 (2005).

6. wwW. Engineeringtoolbox.com [cited 14.02.12]. 